\title{
Low Dispersion Integrated Michelson Interferometer on Silicon on Insulator for Optical Coherence Tomography
}

\author{
Gunay Yurtsever ${ }^{*}$, Katarzyna Komorowska, Roel Baets \\ Ghent University - IMEC, Photonics Research Group, Department of Information Technology (INTEC) \\ ,Sint-Pietersnieuwstraat 41, 9000 Gent, Belgium \\ gunay@intec.ugent.be
}

\begin{abstract}
We present an integrated silicon Michelson interferometer for OCT fabricated with wafer scale deep UV lithography. Silicon waveguides of the interferometer are designed with GVD less than $50 \mathrm{ps} / \mathrm{nm} . \mathrm{km}$. The footprint of the device is $0.5 \mathrm{~mm} \times 3$ $\mathrm{mm}$. The effect of sidewall roughness of silicon waveguides has been observed, possible solutions are discussed.
\end{abstract}

Keywords: optical coherence tomography, silicon photonics, integrated photonics, Michelson interferometer

\section{INTRODUCTION}

Current OCT systems are constructed from fiber/free space optics components. Although fiber and micro-optical components have made these systems portable, further significant miniaturization and cost reduction could be achieved through use of compact integrated photonic components for OCT. As the microfabrication technology improves, it becomes feasible to realize integrated, low-loss, broadband photonic components that can be useful in OCT systems. Integrated photonic components can be fabricated on different material systems, which have their pros and cons. Previously, Culernann et al. demonstrated a passive photonic circuit for OCT fabricated on silica. The device consisted of cascaded splitters and combiners [1]. The photonic structures in glass are very low loss, transparent in the visible, however, due large bend radii, the size of the components are almost two orders of larger than the ones on silicon. In another study, Margallo-Baas et al. demonstrated an integrated photonic structure on silicon on insulator (SOI) for time domain OCT, where the delay line was implemented with thermal tuning of a waveguide in the reference arm [2]. Recently, Nguyen et al. implemented an OCT with an integrated spectrometer fabricated on SiON [3]. Among those silicon on insulator (SOI) has advantages in terms of integration density and possibility to integrate with electronics.

Nanophotonic silicon on insulator is a versatile platform for wafer scale fabrication of variety of integrated photonic components [3]. An SOI wafer consists of a thin top $\mathrm{Si}$ (refractive index, $\mathrm{n}=3.45$ ) layer sitting on silica $\left(\mathrm{SiO}_{2}, \mathrm{n}=1.45\right)$ layer, which is carried on a thick Si substrate. Photonic components are realized by etching the top Si layer, resulting in high refractive index contrast in all directions. Using wafer scale CMOS processes, low loss $(<2 \mathrm{~dB} / \mathrm{cm})$ waveguides with core sizes of $0.1 \mu \mathrm{m}^{2}$ and bend radii of $5 \mu \mathrm{m}$ can be realized. Using such wafer scale processes for silicon, low-cost, high density, integrated photonic components can be mass-fabricated and integrated with CMOS electronics on the same substrate[4]. Due to absorption of the silicon in the visible range, the components are designed for wavelengths above $1200 \mathrm{~nm}$. Although silicon is not an efficient light emitter and detector in the near infrared wavelengths, active elements can be heterogeneously integrated on top of the silicon wafer [5].

ºunay@intec.ugent.be 


\section{DESIGN}

For the SOI platform described above, single mode operation is satisfied for waveguide widths smaller than $500 \mathrm{~nm}$. A typical waveguide that has been used for single mode operation around $1550 \mathrm{~nm}$ wavelength is $450 \mathrm{~nm}$ wide. A significant issue with those 450nm wide waveguides is the large group velocity dispersion (GVD), as GVD causes decrease in resolution and sensitivity. Although GVD can be engineered by using different cladding materials such as silicon dioxide (oxide), polymer etc., changing the width of the waveguides has a much stronger effect on the GVD. As a polymer cladding we have simulated for benzocyclobutene (BCB), which has a refractive index of 1.54 at $1550 \mathrm{~nm}$. Waveguides that are 585 $\mathrm{nm}$ wide with BCB cladding exhibit sufficiently low GVD, which can be easily compensated numerically.
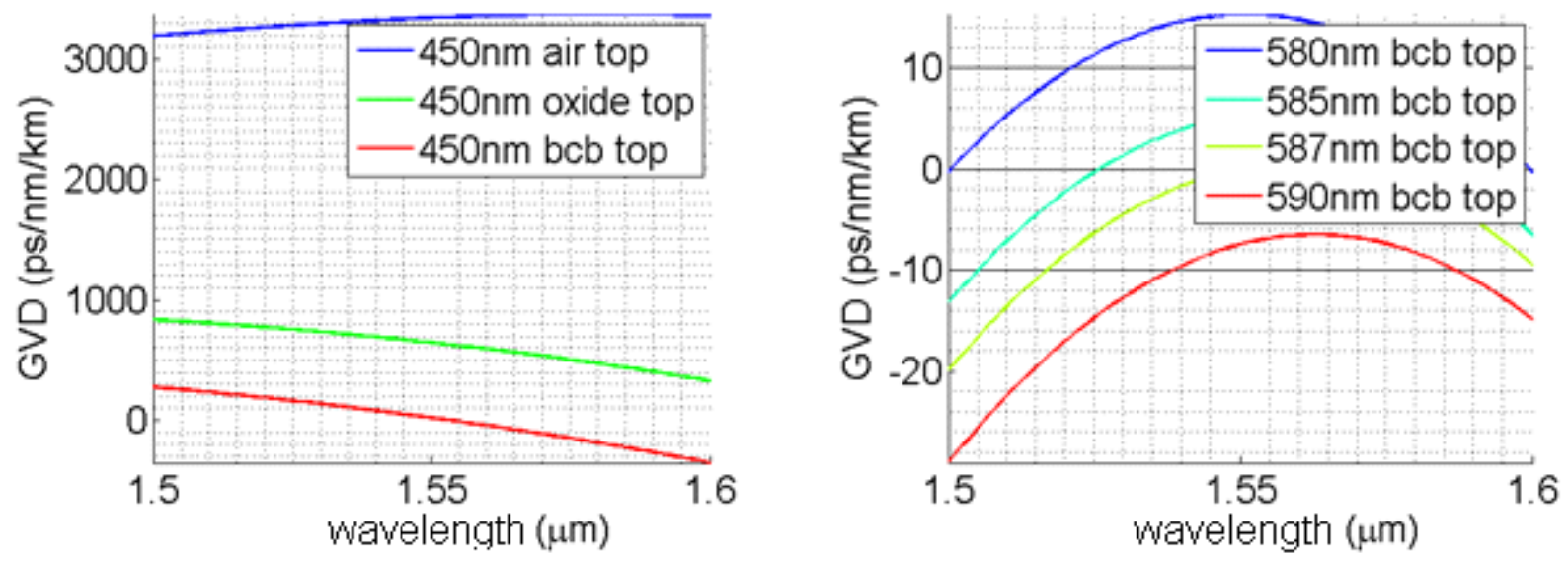

Figure 1. Group velocity dispersion for 450nm (a) and $580 \mathrm{~nm}$ (b) waveguide widths with different top cladding materials.

At 585nm width, those waveguides support two TE modes, which would couple to each other at small waveguide bends, while single mode operation is necessary for optimum interference. Waveguide bends are necessary to have a compact reference arm in spiral shape. We address this problem, by adiabatically tapering down the $585 \mathrm{~nm}$ wide waveguides down to $450 \mathrm{~nm}$ just before the bends, and adiabatically taper back to $585 \mathrm{~nm}$ after the bends.

We have designed and fabricated a Michelson interferometer on SOI using 585nm waveguides. The device is illustrated in Figure 2. A tunable laser centered at $1550 \mathrm{~nm}$ is coupled to the planar photonic chip via a grating coupler which has a 3dB bandwidth of $46 \mathrm{~nm}$ and 25\% coupling efficiency. The $3 \mathrm{~dB}$ bandwidth of the grating coupler can be increased to $80 \mathrm{~nm}$, and coupling efficiency to $70 \%$ by an extra processing step[6]. The size of the grating coupler is $10 \mu \mathrm{m}$ and is tapered to a single mode waveguide.

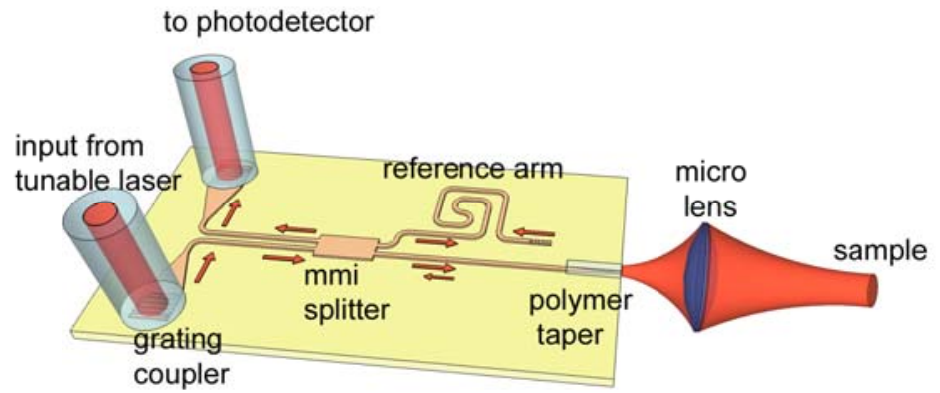

Figure 2. Illustration of the integrated Michelson Interferometer for OCT. 
The waveguides are etched $220 \mathrm{~nm}$ deep and $585 \mathrm{~nm}$ wide after patterning by deep UV lithography. Light is split into reference and sample arms with a 50/50 broadband multimode interference splitter ( $>100 \mathrm{~nm}$ bandwidth at $3 \mathrm{~dB}$ ). The insertion loss of the splitter is less than $1 \mathrm{~dB}$. The reference arm consists of a $9.6 \mathrm{~mm}$ long spiral waveguide, which ends with a silicon air interface. Ideally, the waveguide - air interface acts as a broadband reflector with $30 \%$ reflection efficiency. In the sample, the light is coupled out of the chip with an SU8 polymer spot size converter and is focused onto the sample with a micro lens. The reflections from the sample and reference arms are combined with the splitter and coupled to a fiber which guides the light to a photodiode.

\section{FABRICATION}

An image of the fabricated structure is given in Figure 3. For clarity, only the area around the splitter is shown. The footprint of the device is $0.5 \mathrm{~mm} \times 3 \mathrm{~mm}$. The device is fabricated on a $200 \mathrm{~mm}$ SOI wafer with $220 \mathrm{~nm}$ thick Si top layer on top of 2 $\mu \mathrm{m}$ thick $\mathrm{SiO}_{2}$ using 193nm deep-UV lithography in combination with inductively coupled plasma reactive ion etching. The fabrication was done through the ePIXfab silicon photonics platform at IMEC, Belgium.

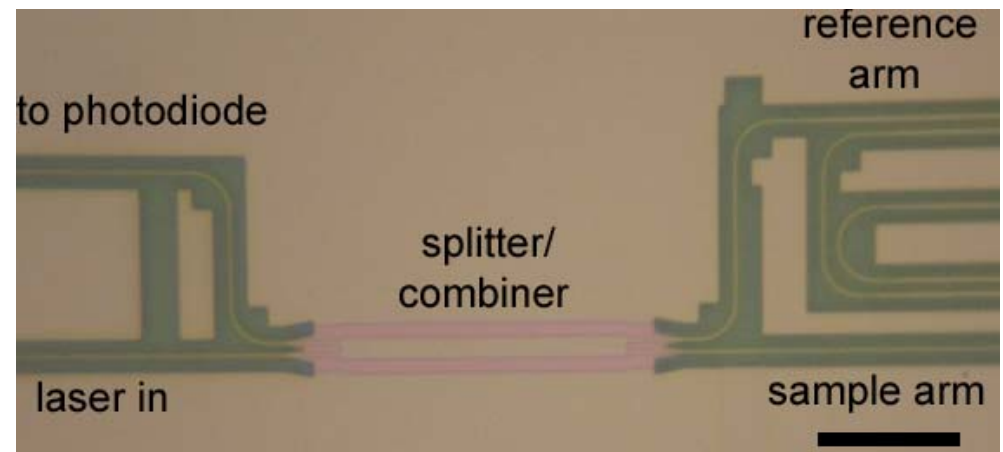

Figure 3. A light microscope image of the center area of the fabricated structure. The scale bar is 20 microns

Broadband out-coupling from an SOI chip can be achieved by cleaving the sample arm waveguide and polishing the waveguide facet. However, coupling out directly from a small silicon waveguide has disadvantages such as requirement for a high NA lens, reduced collection efficiency and sensitivity to lens alignment. An alternative approach for broadband outcoupling is to use a spot size converter [7]. A spot size converter gradually transforms a highly confined waveguide mode into a larger mode supported by a low index contrast waveguide (such as polymers or oxides). Representation of a polymer spot size converter is given in Figure 4a. The silicon waveguide is tapered to a narrower tip, which adiabatically pushes the mode out of the silicon waveguide and couples to the fundamental mode of the polymer waveguide. As a polymer we used SU8 (MicroChem Corp., USA), a photodefinable polymer with refractive index of 1.575 at $1550 \mathrm{~nm}$. We fabricated the polymer waveguide using spin coating and photolithography. After the definition of the polymer waveguide with photolithography, part of the polymer waveguide was cleaved to create a flat facet. A scanning electron microscope image of the SU8 waveguide facet is shown in figure 3b. In our design, the $585 \mathrm{~nm}$ Si waveguide is tapered down to $100 \mathrm{~nm}$ width. The taper length of the Si waveguide was $350 \mu \mathrm{m}$. The fabricated SU8 spot size converter was $3 \mu \mathrm{m}$ wide and $1.6 \mu \mathrm{m}$ high. The insertion loss of the polymer waveguide was $1-1.5 \mathrm{~dB}$. The grating couplers can also be replaced by spot size converters if a very broad band operation is desired. 


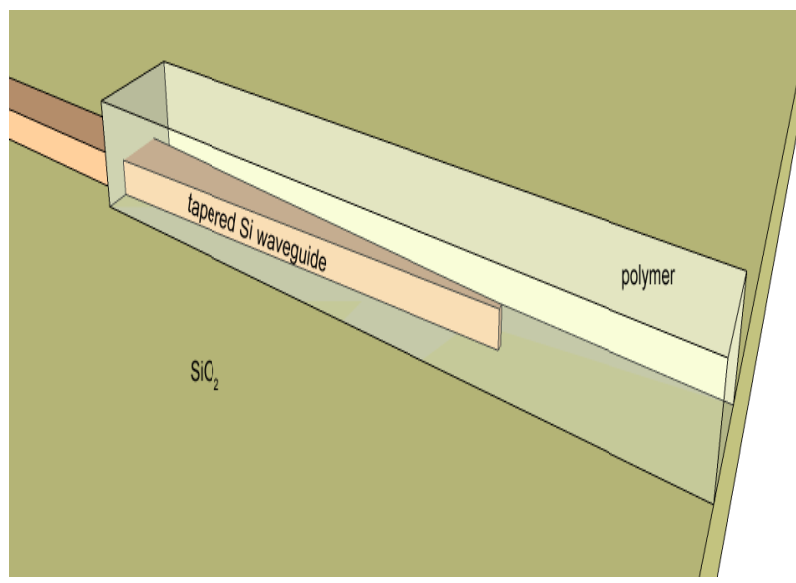

Figure 3a. There dimensional representation of the spot size converter.

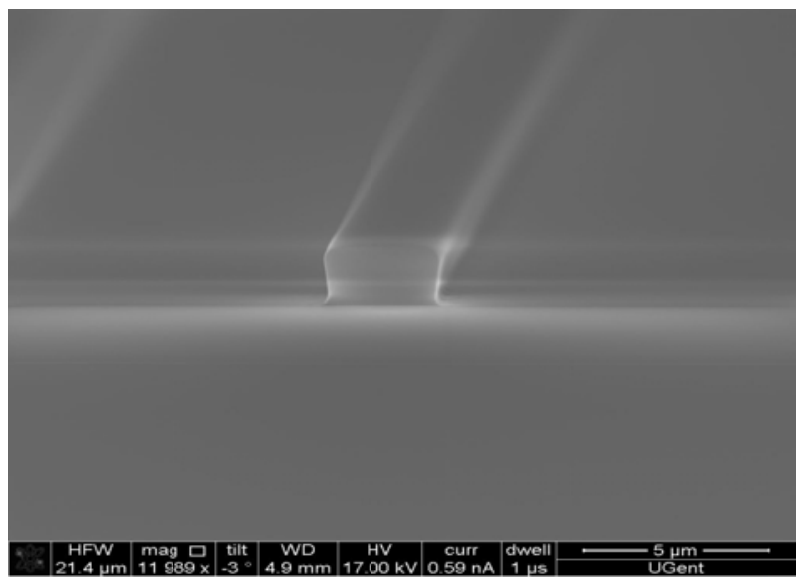

Figure 3b Scanning electron microscope image of the polymer waveguide facet.

\section{MEASUREMENTS}

Dispersion of the waveguides were characterized using separate Mach-Zehnder interferometers with 450nm and $585 \mathrm{~nm}$ waveguides [8]. The results are given in Figure 4. For $450 \mathrm{~nm}$ wide waveguides BCB polymer cladding significantly reduces the GVD as shown in Figure 4a, while GVD of the $585 \mathrm{~nm}$ waveguides with BCB cladding is very small as expected from simulation results (Figure $4 \mathrm{~b}$.

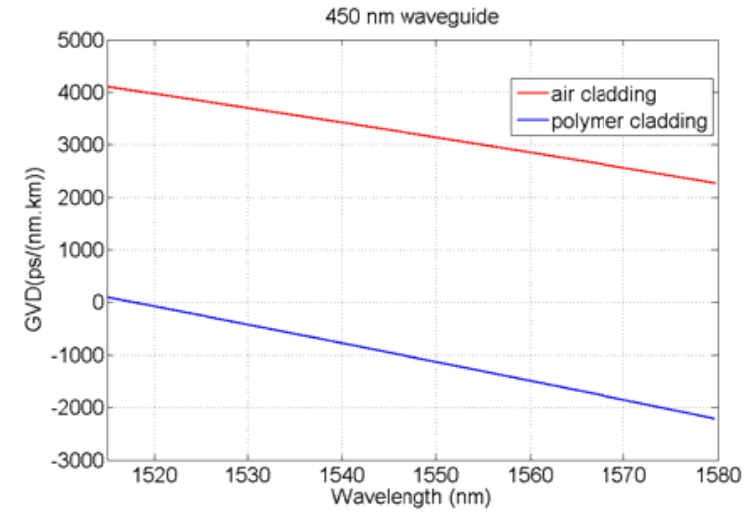

(a) $450 \mathrm{~nm}$ wide waveguide (red: air cladding, blue: BCB cladding).

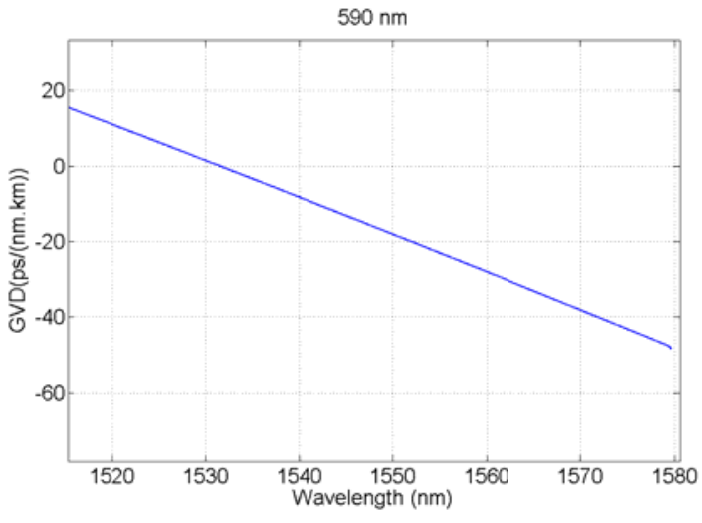

(b) $585 \mathrm{~nm}$ wide waveguide with BCB cladding

Figure 4. Measured group velocity dispersion..

The fabricated Michelson interferometer was characterized without a lens and a sample. As a light source we used a high coherence tunable laser with a tuning rage of $1510 \mathrm{~nm}-1630 \mathrm{~nm}$ (TSL 510, Santec, Japan). The polarization of the light was optimized using a fiber polarization controller for efficient coupling. Light was coupled with a single mode fiber (SMF-28) through the input grating coupler. If there is no sample present, ideally we would expect to see interference of the light reflecting from the reference arm end and the sample arm end. Thus we should see a clear peak at the arm mismatch distance. After sampling the data evenly in frequency and taking Fourier Transform we obtained the reflection profile given in Figure 5. The reference arm length is $9.6 \mathrm{~mm}$ and the sample arm length is $2.2 \mathrm{~mm}$. The peak at $9.6 \mathrm{~mm}$ is the interference of 
reflections from the reference arm and the MMI splitter, while the peak at $7.4 \mathrm{~mm}$ is the interference of reflections from the reference arm end and the sample arm end.

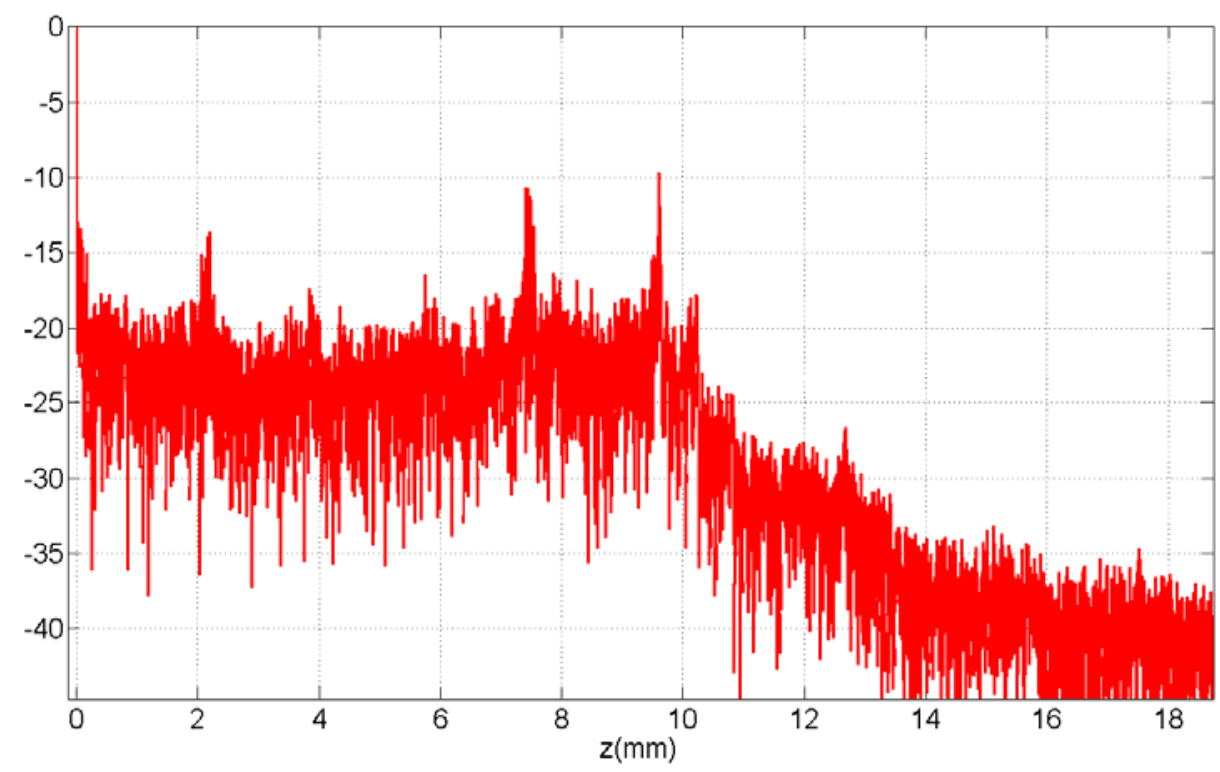

Figure 5. Reflection profile of the Michelson interferometer without sample.

The noise level is unexpectedly high that imaging a scattering sample was not attempted. The high noise level is due to backscattering in the waveguides. Thus the waveguides acts as a distributed reflector. The sidewall roughness of the waveguides induces significant backscattering [9]. In this study, the waveguides were defined using dry etching which is known to create side wall roughness. The problem of side wall roughness can be eliminated by defining the silicon waveguides by thermal oxidation [10]. Also, rib waveguides with less sensitivity to side wall roughness can be used instead of the current ridge waveguides. Another solution can be using a Mach-Zehnder interferometer rather than Michelson interferometer.

\section{CONCLUSION}

We have designed and fabricated a Michelson interferometer using integrated silicon waveguides. Using a polymer cladding low dispersion waveguides have been realized. The side wall roughness induced backscattering in the waveguides poses a problem to use such an interferometer for OCT. However, the side wall roughness can be solved using thermal oxidation to define the waveguides.

Silicon photonics is maturing as telecom applications demand more complex and high performance integrated photonics circuits. OCT can potentially benefit from those advances for more compact and low cost devices. 


\section{REFERENCES}

[1] Culernann D, Knuettel A, Voges E. "Integrated optical sensor in glass for optical coherence tomography”, IEEE Journal of Selected Topics in Quantum Electronics, 5(6), p. 730-734 (2000).

[2] Margallo-Baas E, Pandraud G, French PJ. "Thermo-Optical Delay Line for Miniature Optical Coherence Tomography”, Proceedings of the Conference on Coherence Domain Optical Methods and Optical Coherence Tomography in Biomedicine XII,vol6847, p. S8470- S8470 (2008).

[3] Nguyen VD, Akca BI, Wörhoff K, et al. "Spectral domain optical coherence tomography imaging with an integrated optics spectrometer”. Optics letters, 36(7), p.1293 (2011).

[4] Bogaerts W, et al. "Nanophotonic waveguides in silicon-on-insulator fabricated with CMOS technology" Journal of Lightwave Technology, 23(1), p. 401-412 (2005).

[5] Roelkens $\mathrm{G}$ et al. "Light emission and enhanced nonlinearity in nanophotonic waveguide circuits by III-V/SOI heterogeneous integration”. Journal of Applied Physics, 104(3), p.033117 (2008).

[6] Vermeulen D, Selvaraja S, Verheyen P, Lepage G, Bogaerts W, Absil P, Van Thourhout D, Roelkens G. "High-efficiency fiber-to-chip grating couplers realized using an advanced CMOS-compatible silicon-on-insulator platform”, Optics Express, 18(17), p.18278-18283 (2010).

[7] Shoji T, Tsuchizawa T, Watanabe T, Yamada K, Morita H. "Low loss mode size converter from $0.3 \mu \mathrm{m}$ square Si wire waveguides to singlemode fibres”, Electronics Letters, 38(25), p.1669-1670 (2002).

[8] Dulkeith E, Xia F, Schares L, et al. "Group index and group velocity dispersion in silicon-on-insulator photonic wires" Optics express.14(9), p.3853-3863 (2006)

[9] Morichetti F et al. "Roughness Induced Backscattering in Optical Silicon Waveguides”. Physical Review Letters, 104(3), p.1-4 (2010)

[10] Cardenas J, Poitras CB, Robinson JT, et al. “Low loss etchless silicon photonic waveguides”. Optics Express. 17(6), p.4752-7 (2009). 